\title{
Flow pattern of accelerated cometary ions inside and outside the diamagnetic cavity of comet 67P/Churyumov-Gerasimenko
}

\author{
K. Masunaga ${ }^{1}$, H. Nilsson ${ }^{1}$, E. Behar ${ }^{1}$, G. Stenberg Wieser ${ }^{1}$, M. Wieser ${ }^{1}$, and C. Goetz ${ }^{2}$ \\ ${ }^{1}$ Swedish Institute of Space Physics, Box 812, Kiruna 981 28, Sweden \\ e-mail: kei@irf.se \\ ${ }^{2}$ Institut für Geophysik und extraterrestrische Physik, TU Braunschweig, Mendelssohnstr. 3, 38106 Braunschweig, Germany
}

Received 24 January 2019 / Accepted 7 June 2019

\begin{abstract}
Analyzing data from the Ion Composition Analyzer on board the Rosetta spacecraft, we studied a flow pattern of accelerated cometary ions $(40-80 \mathrm{eV}$ ) inside and outside the diamagnetic cavity of comet 67P/Churyumov-Gerasimenko (67P). We found that the accelerated ions are intermittently observed and are ten times more frequently observed outside the cavity than inside, and they mainly flow tailward with an aberration $\left(\sim 20-40^{\circ}\right)$. We suggest that they are accelerated by the tailward polarization electric field upstream of the comet. Because their occurrence frequency becomes lowest near perihelion where the water production rate is highest at 67P, ion-neutral collisions and/or charge exchange may play a role in controlling the occurrence frequency. The aberration pattern is different inside and outside the cavity in the cometocentric solar equatorial (CSEQ) frame but it is consistent in the comet-Sun electric (CSE) frame; the latter is rotated from the CSEQ frame about the comet-Sun line so that the $Z$-axis is aligned with the local motional electric field. Because the flow pattern of the accelerated ions inside the cavity in the CSE frame is the same as outside, we suggest that the flow pattern inside is determined by the flow outside, depending on the local plasma and magnetic field. Near the CSE polar plane the aberration is in the opposite direction of the motional electric field, while it is in the anti-cometward direction near the CSE equator plane. The aberration in the anti-electric-field direction near the CSE polar plane suggests that the accelerated ions are mass-loaded by local cold cometary ions, just like the mass-loading of the solar wind by cold cometary ions. The cause of the anti-cometward aberration near the CSE equator plane is still unknown, but this may indicate that the tailward-flowing cometary ions are deflected across the upstream boundaries or by an outward-pointing ambipolar electric field.
\end{abstract}

Key words. comets: general - comets: individual: 67P/Churyumov-Gerasimenko - plasmas - magnetic fields methods: observational

\section{Introduction}

Comets are small (typically a few to $10 \mathrm{~km}$ ) rocky and icy objects that originate from the Kuiper belt or the Oort cloud. Some of them periodically orbit the Sun, but others pass the Sun by once and do not return. When a comet approaches the Sun, volatiles near its surface are heated and sublimated. This creates an atmosphere around the comet, a so-called coma. It usually extends millions of kilometers around a comet because the gravity of the comet is too weak to keep the atmosphere.

The cometary coma is composed of dusts and neutral gas originating from the nucleus. The dust particles are typically microscale grains that are pushed away from the coma by the solar radiation pressure, resulting in the formation of a dust tail behind the comet. The neutral gas in the coma is partially ionized by solar extreme ultraviolet (EUV) radiation, electron impact ionization, or charge exchange with the solar wind. When the solar wind meets the partially ionized atmosphere, the new-born ions are accelerated by the solar wind motional electric field and are picked up by the solar wind (e.g., Coates 2004). Because the gyroradii of cometary pickup ions are larger than the size of the comet nucleus, the cometary pickup ions near the comet generally flow along the motional electric field. At the same time, the mass of the pickup ions is loaded into the solar wind, which causes the bulk plasma speed to slow down. Then the solar wind ions (mainly protons) start to feel the Lorentz force of the interplanetary magnetic field (IMF) frozen into the bulk plasma and are deflected from the Sun-comet line in the opposite direction of the cometary pickup ions. These processes are referred to as the mass-loading of the solar wind (e.g., Szegö et al. 2000; Behar et al. 2016a).

Rosetta was the first spacecraft that orbited a comet (Glassmeier et al. 2007a). It was launched in 2004 and arrived at comet 67P/Churyumov-Gerasimenko (67P) in August 2014. Multiple instruments of the Rosetta Plasma Consortium (RPC; Carr et al. 2007) collected in situ plasma and field measurements around 67P until September 2016. Using the Ion Composition Analyzer (ICA; Nilsson et al. 2007), a part of the RPC, a weak mass-loading of the solar wind was observed around 67P at the beginning of the mission in 2014 (Nilsson et al. 2015; Behar et al. 2016a). Behar et al. (2016b, 2017) later studied the evolution of the mass-loading at 67P. They found that the deflection angle of the solar wind protons increased as the comet outgassing rate increased. As the outgassing rate became even higher near perihelion, the solar wind protons were eventually deflected up to $180^{\circ}$ from the Sun-comet line. The proton gyro motion forms a finite region around the comet that the protons do not enter. This region is called the solar wind ion cavity (SWIC; Behar et al. 2017).

Although there are no solar wind protons in the solar wind cavity, the IMF is still carried inward by the electron fluid of the solar wind. Owing to the decreasing bulk velocity of the fluid 
in this region after a significant mass-loading, the IMF starts to pile up and eventually stops to form a boundary inside which the magnetic field becomes zero (Goetz et al. 2016a,b). This region is referred to as the diamagnetic cavity.

The existence of the diamagnetic cavity was theoretically predicted by Biermann et al. (1967). It was later directly observed by the Giotto spacecraft at comet 1P/Halley (Neubauer et al. 1986). The distance to the boundary was $4760 \mathrm{~km}$ (inbound) and $3840 \mathrm{~km}$ (outbound) from the comet, which was larger than expected from a simple pressure balance calculation. Cravens (1987) and Ip \& Axford (1987) later suggested that collisions are important inside the diamagnetic cavity and that the ions are strongly coupled with neutrals there because the outward velocities of neutrals $\left(\sim 1 \mathrm{~km} \mathrm{~s}^{-1}\right)$ and ions $\left(\sim 0.9 \mathrm{~km} \mathrm{~s}^{-1}\right)$ were found to be similar inside the diamagnetic cavity.

During the two-year observations of the magnetometer on board Rosetta (MAG, Glassmeier et al. 2007b), the diamagnetic cavity was observed more than 600 times around 67P (Goetz et al. 2016a,b). These observations occurred between April 2015 and February 2016 at a heliocentric distance of $\sim 1.2-2.5$ AU. The distance of the boundary was $\sim 40-380 \mathrm{~km}$ from the nucleus, which means that the size of the diamagnetic cavity of $67 \mathrm{P}$ is much smaller than that of comet Halley. This was expected as the outgassing rate is lower at 67P than at comet Halley. Goetz et al. (2016b) also showed that the diamagnetic cavity of 67P has a dynamic boundary rather than a stable one.

Observations by the Langmuir probe instrument (LAP, Eriksson et al. 2007) on board Rosetta showed that cold cometary ions are moving outward with $\sim 2-4 \mathrm{~km} \mathrm{~s}^{-1}$ inside the diamagnetic cavity, which is slightly faster than the neutrals (Odelstad et al. 2018). Odelstad et al. (2018) suggested that the collisional coupling is not important around 67P, at least at the distances probed by Rosetta, and that the ions are accelerated radially outward from the nucleus by the ambipolar electric field near the nucleus, as also suggested by Berčič et al. (2018).

Observations by the Mutual Impedance Probe (MIP; Trotignon et al. 2007) showed that the plasma density inside the diamagnetic cavity was rather steady on the timescale of individual (or indeed multiple successive) diamagnetic cavity observations. The density varied for different cavity observations between 100 and $1500 \mathrm{~cm}^{-3}$ (Henri et al. 2017). However, a sudden short increase in plasma density, a plasma blob, sometimes appeared inside the cavity (Hajra et al. 2018). It was suggested that the plasma blobs are transferred from the outside of the diamagnetic cavity where the plasma is magnetized. This would mean that there must be a mechanism to transport plasma across the boundary of the diamagnetic cavity.

Accelerated ions $(>40 \mathrm{eV})$ were also observed around $67 \mathrm{P}$ near the time period when the diamagnetic cavity was observed (Stenberg Wieser et al. 2017). Although cold cometary ions flow radially outward at least inside the diamagnetic cavity (Odelstad et al. 2018), Stenberg Wieser et al. (2017) showed cases where the ion energy, flux, and flow direction changed quickly with time. They studied only a few cases, however, and the statistical properties of ions in the energy range $>40 \mathrm{eV}$ are still unknown.

In order to extend our understanding of the accelerated ions near the diamagnetic cavity, in this paper we study characteristics of the accelerated ions in and around the diamagnetic cavity of 67P. Using one of the high time-resolution modes of the ICA, we statistically examine occurrence frequencies of the accelerated ions and also their flow direction in and around the diamagnetic cavity.

\section{Instruments}

We analyzed data obtained from the ICA instrument (Nilsson et al. 2007). In the nominal observation mode, the ICA had a capability to observe ions with energies ranging from a few $\mathrm{eV} \mathrm{q}^{-1}$ to $40 \mathrm{keV} \mathrm{q}^{-1}$ with an energy resolution ( $\left.\frac{\mathrm{d} E}{E}\right)$ of 0.07 . There were 96 energy steps, and it took $12 \mathrm{~s}$ to complete the full energy scan. The field of view of ICA was $90^{\circ} \times 360^{\circ}$ with a resolution of $5.6^{\circ} \times 22.5^{\circ}$ ( 16 elevations and 16 azimuthal sectors), although a part of this field of view was blocked by the spacecraft. By repeating the energy scan for each elevation, a full 3D energy distribution was produced, which took $192 \mathrm{~s}$ in total.

In this study, we investigate the characteristics of the cometary ion energy distribution, which dynamically changes in and around the diamagnetic cavity, by using one of the high timeresolution modes of ICA. By reducing the number of energy steps to 32 and fixing the elevation step to as close as possible to the instrument plane (Nilsson et al. 2007), a $4 \mathrm{~s}$ resolution of $2 \mathrm{D}$ measurements can be achieved. Using the currently best estimate of the energy offset of the ICA relative to the initial revised energy scale (Nilsson et al. 2015), which has been estimated to be $13.7 \mathrm{eV}$ by Odelstad et al. (2017), its energy range in this mode becomes $0.3-82 \mathrm{eV} \mathrm{q}^{-1}$, and the time resolution of the full energy scan becomes $4 \mathrm{~s}$. We thus obtain 2D energy distributions with a field of view of $5.6^{\circ} \times 360^{\circ}$ every $4 \mathrm{~s}$. A more detailed description of the high time-resolution modes can be found in Stenberg Wieser et al. (2017). We focus on cometary ions with energies of $40-80 \mathrm{eV}$ in this study because lower energy ions are strongly affected by the negative spacecraft potential that was typical of Rosetta throughout the mission. We also only use data in a period when Rosetta was usually in the SWIC (i.e., from 28 April, 2015 to $11 \mathrm{Dec}, 2015$ Behar et al. 2017) so we can avoid a contamination from solar wind ions. In this time period, the comet approached the Sun from 1.63 to $1.24 \mathrm{AU}$ at perihelion on 13 August 2015 and then retreated to 1.78 AU.

We also use data obtained from the MAG instrument (Glassmeier et al. 2007b). MAG consisted of two identical triaxial fluxgate sensors and measured the magnetic field in a range of $\pm 16384 \mathrm{nT}$ with a resolution of $31 \mathrm{pT}$. Its sampling rate was nominally $20 \mathrm{~Hz}$, which is much faster than the time resolution of the ICA. The magnetic field data were calibrated as described in Goetz et al. (2016b).

To obtain water production rates at $67 \mathrm{P}$, we use the Haser model (Haser 1957) and in situ measurements of the Cometary Pressure Sensor (COPS) instrument of the Rosetta Orbiter Spectrometer for Ion and Neutral Analysis (ROSINA; Balsiger et al. 2007). In this model we assumed the neutral velocity of $500 \mathrm{~m} \mathrm{~s}^{-1}$. We also use the empirical model of the water production rate at 67P from Hansen et al. (2016) as a basis for comparison.

\section{Observations}

Figure 1 shows the time series of ion and magnetic field observations of three crossings of the diamagnetic cavity on November 20, 2015. The gray dashed vertical lines indicate the crossings of the diamagnetic cavity boundary identified from the magnetic field shown in Fig. 1d (Goetz et al. 2016a,b). Figure 1a is an ion energy spectrogram observed in the ICA high time-resolution mode. The lower energy cutoff is caused by a negative spacecraft potential that accelerated the ions into the instrument (Odelstad et al. 2017; Stenberg Wieser et al. 2017). An orange curve shows this lower cutoff. Figure $1 \mathrm{~b}$ shows the same ion energy spectra as Fig. 1a, but its energy is corrected 


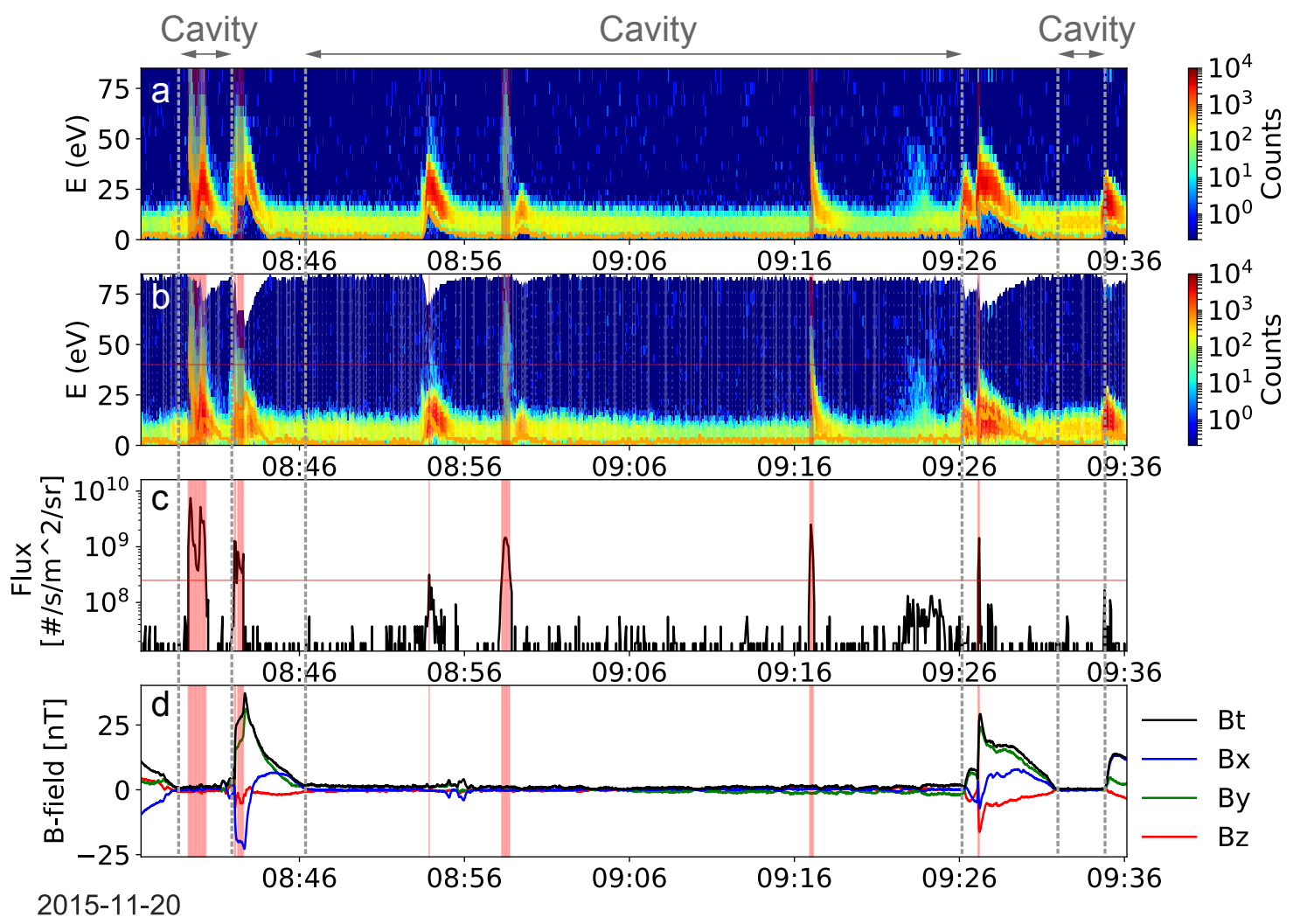

Fig. 1. (a) original ion energy-time spectrum, $(b)$ ion energy-time spectrum corrected for the spacecraft potential, $(c)$ average ion fluxes (40-80 eV), and $(d)$ magnetic field. The gray vertical dashed lines indicate the boundaries of the diamagnetic cavity. The red vertical lines indicate detections of accelerated ions at the respective time stamps per energy scan.

for the spacecraft potential. This figure shows that the ICA constantly observed very low energy cometary ions below $25 \mathrm{eV}$ but sometimes observed accelerated ions (several tens of eV) for a short time period. The accelerated ions usually show dispersive energystructures and often coincide with steepened magnetic field surrounding the cavity, which generally corresponds to the characteristics seen in types 4 or 5 studied in Stenberg Wieser et al. (2017). We did not observe the solar wind ions in this time period because Rosetta was in the SWIC, which is much larger than the diamagnetic cavity (Behar et al. 2017). We developed a method for detecting these accelerated ions automatically. Figure 1c presents the average ion fluxes integrated over the energy range between 40 and $80 \mathrm{eV}$ corrected for the spacecraft potential. We used a lower limit of the average ion fluxes to define a detection of the accelerated ions: $2.5 \times 10^{8} \mathrm{~s}^{-1} \mathrm{~m}^{-2} \mathrm{sr}^{-1}$ as shown by the red horizontal line in this figure. When the average flux was higher than this value, we considered this a detection of the accelerated ions. As indicated by red vertical lines, the accelerated ions are reasonably well detected using this criterion.

We applied the automatic selection method to the 79 diamagnetic cavity observations where ICA was operating in the high time-resolution mode. The time period spent inside the diamagnetic cavity was different for each cavity crossing. Outside the diamagnetic cavity we restricted our search for accelerated ions to $10 \mathrm{~min}$ from the inbound and outbound boundaries. If two or more cavity crossings were observed close to each other, as shown in Fig. 1, then the time period of the outside ends at the previous (next) outbound (inbound) boundary and thus becomes shorter than $10 \mathrm{~min}$. The second columns (shown as "All") of Tables 1 and 2 show the time in total for which the accelerated ions were detected outside and inside the diamagnetic cavity. This time was compared to the total observation time, and the occurrence frequency of the accelerated ion detections was calculated, which are also given in these tables. The occurrence frequency outside the cavity is ten times higher than inside the cavity (18\% outside the cavity and $1.8 \%$ inside the cavity).

Figures 2a,b show the time series of the heliospheric distance of 67P when Rosetta was just outside and inside the cavity, respectively. The blue and gray markers in these figures indicate whether Rosetta observed the accelerated ions at the timestamps. Figure 2c shows the time series of the water production rate at 67P obtained from the Haser model (Haser 1957) using ROSINA/COPS measurements (red dots) and the empirical model of Hansen et al. (2016; black line). Based on the heliospheric distances and water production rates, we divided the observation period into three subperiods $\mathrm{P} 1, \mathrm{P} 2$, and $\mathrm{P} 3$, as indicated above the top panel in this figure. We calculated the occurrence frequency of the accelerated ions in each subperiod as shown in Tables 1 and 2. The median production rates were also calculated using our observation time-stamps in each subperiod, and they are shown in Table 3 . The occurrence frequency of the accelerated ions decreases as the production rate increases both outside and inside the cavity. On the other hand, the ratio of the occurrence frequency between inside and outside the cavity remains the same throughout the three subperiods. The occurrence frequency outside the cavity is nearly ten times higher than inside the cavity in each subperiod.

Figure 3 shows the positions of Rosetta where the accelerated ions are either detected or not detected outside the cavity. Figures 3a,b show the positions in the CSEQ frame where the $X$-axis points to the Sun, the $Z$-axis is parallel to the solar 

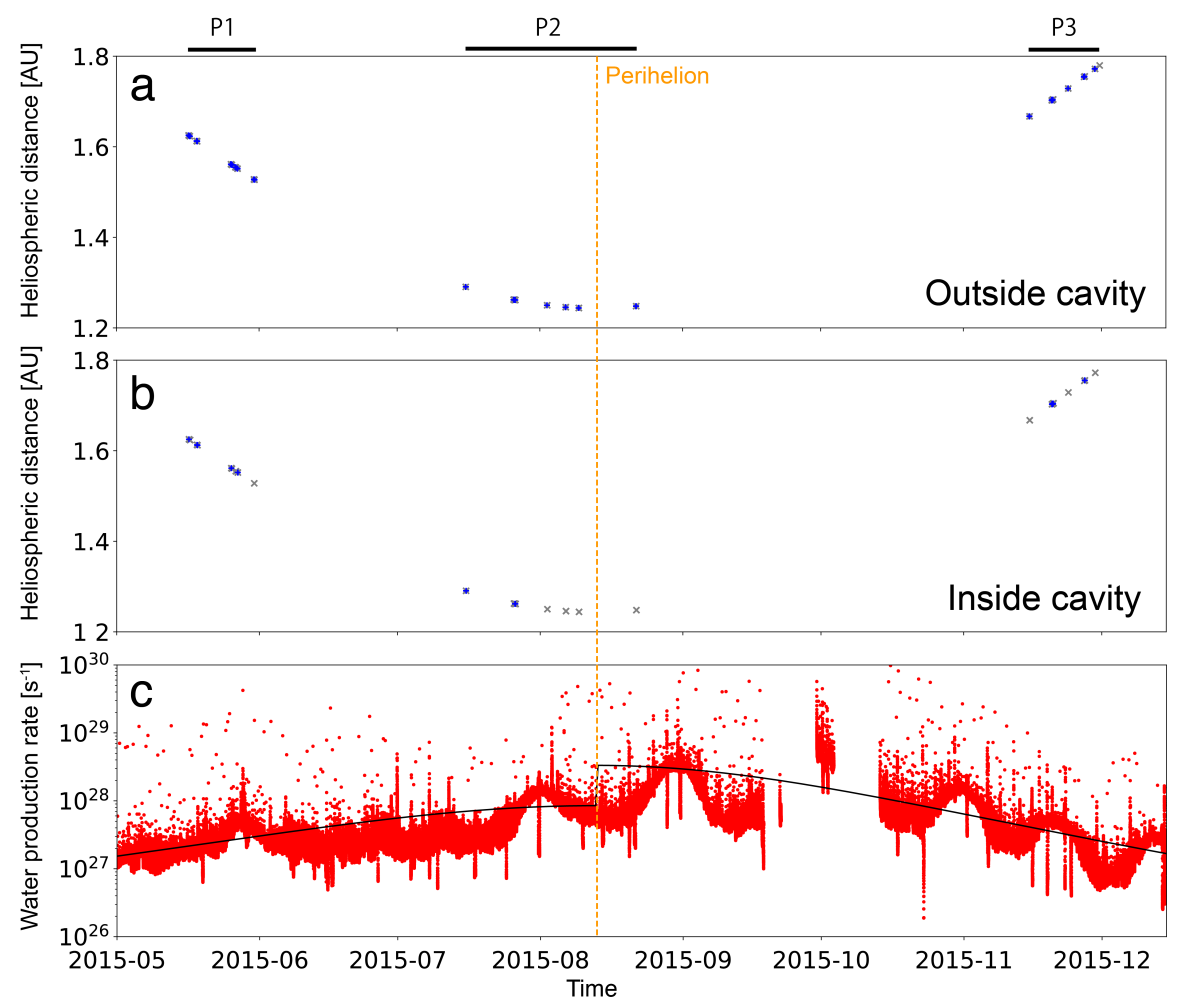

Fig. 2. Time series of the heliospheric distance of 67P during which Rosetta was $(a)$ just outside and $(b)$ inside the cavity. The blue and gray markers indicate times when the accelerated ions were or were not observed. (c) Time series of the water production rate at 67P obtained from the Haser model using ROSINA/COPS observations (red dots) and the Hansen model (black). P1, P2, and P3 shown above the figure are three subperiods of our observation period.

Table 1. Duration of accelerated ions' detection, the total observation time, and their occurrence frequency outside the diamagnetic cavity for our all observation period and three different subperiods.

\begin{tabular}{ccccc}
\hline \hline Outside cavity & All & P1 & P2 & P3 \\
\hline Detection of accelerated ions (min) & 165 & 85.7 & 22.1 & 56.7 \\
(Number of time stamps) & $(2468)$ & $(1285)$ & $(332)$ & $(851)$ \\
Total observation time (h) & 15.4 & 3.23 & 7.29 & 4.91 \\
(Number of time stamps) & $(13889)$ & $(2907)$ & $(6562)$ & $(4420)$ \\
Occurrence frequency (\%) & 18 & 44 & 5 & 19 \\
\hline
\end{tabular}

Notes. Number of timestamps corresponding to each duration is shown in brackets.

Table 2. Duration of accelerated ions' detection, the total observation time, and their occurrence frequency inside the diamagnetic cavity, which is the same format as Table 1.

\begin{tabular}{ccccc}
\hline \hline Inside cavity & All & P1 & P2 & P3 \\
\hline Detection of accelerated ions (min) & 6.46 & 0.60 & 0.47 & 5.40 \\
(Number of time stamps) & $(97)$ & $(9)$ & $(7)$ & $(81)$ \\
Total observation time (h) & 5.97 & 0.27 & 1.48 & 4.21 \\
(Number of time stamps) & $(5373)$ & $(247)$ & $(1335)$ & $(3791)$ \\
Occurrence frequency (\%) & 1.8 & 3.6 & 0.5 & 2.1 \\
\hline
\end{tabular}

Table 3. Median water production rate in each subperiod.

\begin{tabular}{cccc}
\hline \hline Subperiod & P1 & P2 & P3 \\
\hline Median water production rate $\left(10^{27} \mathrm{~s}^{-1}\right)$ & 3.4 & 6.1 & 4.9 \\
\hline
\end{tabular}

rotational axis, and the $Y$-axis completes the right-hand system. Figures $3 \mathrm{c}, \mathrm{d}$ are shown in the CSE frame where the $X$-axis points to the Sun, the $Y$-axis along the local magnetic field component in the $Y Z$ plane, and the $Z$-axis completes the right-handed system. To convert the positions from the CSEQ frame into the CSE frame, we used the average local magnetic field outside the cavity in each cavity crossing. No solar wind ions were recorded locally because Rosetta was inside the SWIC (Behar et al. 2017), but because the clock angle of the magnetic field in the upstream solar wind tended to remain the same in the inner coma (Edberg et al. 2019), we can assume a general anti-sunward motion of the magnetic field (bulk plasma) and thus the motional electric field would point upward $\left(+Z_{\mathrm{CSE}}\right)$. Nevertheless, we do not see any asymmetry in the spatial distribution of the accelerated ions in either of the two coordinate systems. The accelerated ions are spread around the comet everywhere.

Figure 4 shows Rosetta positions with detections of accelerated ions or without them inside the cavity in the same format as Fig. 3. The occurrence frequency of the accelerated ions is lower inside the cavity than outside (see Tables 1 and 2), therefore the gray crosses dominate in this figure. Similar to Fig. 3 observed outside the cavity, the positions of the accelerated ions do not show any asymmetric distribution in either the CSEQ or CSE frames.

In order to study the flow direction of the accelerated ions, we statistically analyzed the observed ion fluxes in the different azimuthal sectors of ICA. We discarded sector 0 due to crosstalk effects: sector 0 received a certain percent of counts from other sectors (Berčič 2017), which prevented us from identifying a flow direction. Sector 13 was not used either because it had an extremely low sensitivity and thus almost no counts 

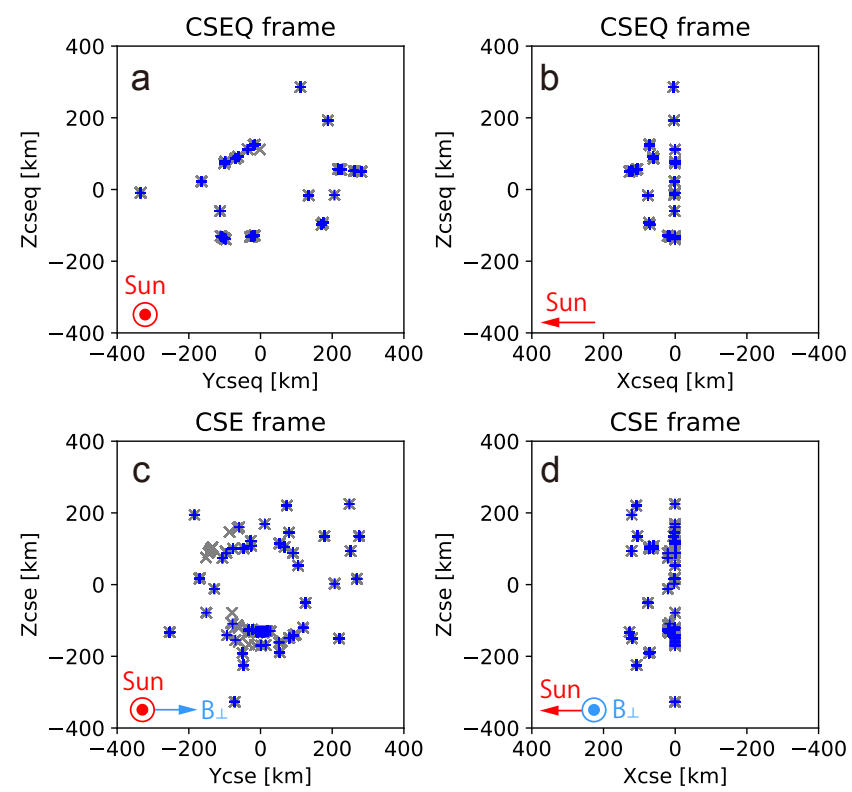

Fig. 3. Rosetta positions when the accelerated ions were observed (blue plus) or were searched for, but were not observed (gray cross) outside the diamagnetic cavity in the CSEQ (top panels) and the CSE (bottom panels) frames. Many markers overlap because of observations with the high time-resolution mode and the slow speed of the spacecraft.

were detected. Because Rosetta usually orbited in the terminator plane and the spacecraft $+Z_{\mathrm{SC}}$ plane faced the comet, the viewing direction of each sector of the ICA was fixed with respect to the Sun and the comet most of the time. For example, sectors 8 or 9 looked sunward and sectors 4 or 5 looked cometward, detecting anti-sunward and anti-cometward flowing ions, respectively. Rosetta, however, was sometimes located out of the terminator plane, as shown in Figs. 3 and 4. In these cases, the viewing direction of the sectors changed. When Rosetta was on the dayside and the $+\mathrm{X}_{\mathrm{SC}}$ plane faced the comet, sector 10 instead of sectors 8 or 9 tended to look at the Sun. When the $+X_{\mathrm{SC}}$ plane faced the Sun on the dayside, sector 3 instead of sectors 4 or 5 looked at the comet. This should be taken into account when an average ion flow pattern with respect to the Sun and comet is to be obtained. In order to do so, we defined a new sector plane with 16 sectors running from $0^{\prime}$ to $15^{\prime}$. In this new sector plane, the Sun-viewing sector was always fixed to sector $9^{\prime}$. Thus, viewing directions of sectors $9^{\prime}$ and $1^{\prime}$ are nearly parallel to the Suncomet line (the $X$-axis of CSEQ and CSE frames). Depending on the Rosetta position and attitude, the comet-viewing sector changed from sector $3^{\prime}$ to $5^{\prime}$. For example, when the Sun- and comet-viewing directions corresponded to the sectors 10 and 4 using the original sector numbering, they would be $9^{\prime}$ and $3^{\prime}$ in the new sector numbering scheme.

Using the new sector plane, we first investigated the average flow pattern of accelerated ions in the CSEQ frame. Because the ICA field of view is a 2D plane in the high time-resolution mode, we also considered the clock angle (CLA) of the Rosetta position, which is the angle measured from the $Y$-axis toward the $Z$-axis. We divided the data into four cases according to the CLA: region (1) $-45^{\circ} \leq \mathrm{CLA} \leq 45^{\circ}$, region (2) $45^{\circ} \leq \mathrm{CLA} \leq$ $135^{\circ}$, region (3) $135^{\circ} \leq \mathrm{CLA} \leq 225^{\circ}\left(-135^{\circ}\right)$, and region (4) $-135^{\circ} \leq \mathrm{CLA} \leq-45^{\circ}$. This allowed us to discuss ion flow directions approximately near the $X Y$ plane for regions 1 and 3 and near the $X Z$ plane for regions 2 and 4 . Figures $5 \mathrm{a}-\mathrm{d}$ show the average energy-sector flux distributions of the accelerated ions
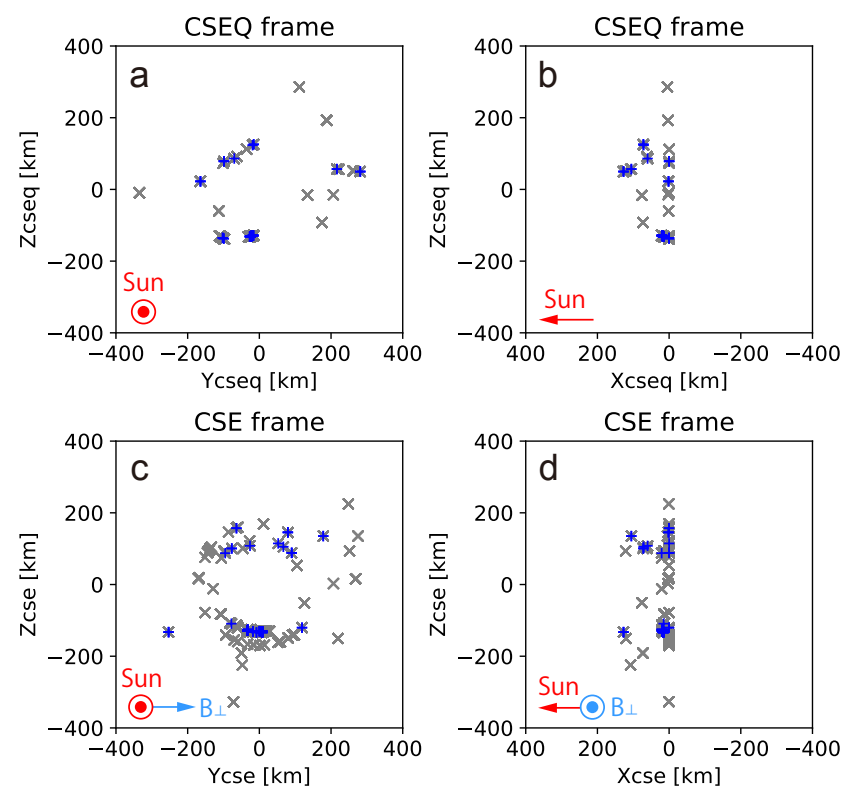

Fig. 4. Rosetta positions when the accelerated ions were observed (blue plus) or searched for but not observed (gray cross) inside the diamagnetic cavity. The format is the same as in Fig. 3.

(40-80 eV) observed outside the diamagnetic cavity in the four regions. The closed red and gray circles by the edge of each plot indicate the average Sun and comet directions within sector 9' and sectors $3^{\prime}, 4^{\prime}$, and $5^{\prime}$, respectively. Strong ion fluxes respectively. Strong ion fluxes appear in sectors $7^{\prime}$ and $8^{\prime}$ in regions 1 , 2 , and 4 and in sectors $10^{\prime}$ and $11^{\prime}$ for region 3 . This means that the accelerated ions mainly flow tailward with a cometward/anticometward aberration $\left(\sim 20-40^{\circ}\right)$ outside the diamagnetic cavity because the ion flow detected in sector $9^{\prime}$ is a pure tailward flow. The energy of the high ion fluxes is around the lower boundary of the energy range, $\sim 40 \mathrm{eV}$. Figures $5 \mathrm{e}-\mathrm{h}$ show the same plots but observed inside the diamagnetic cavity. These flux distributions look generally similar to what we see outside the cavity, but there are some noteworthy differences. The main flow component is tailward in regions $1-3$, but the aberration component is different from that outside the cavity in regions 2 and 3. In region 4 , the highest flux appears in sector $13^{\prime}$, which corresponds to a cometward flow, although the sector distribution looks similar to that of the outside. In addition, the flux of the accelerated ions is nearly one order of magnitude lower inside the cavity than outside the cavity.

Figure 6 shows the average energy-sector flux distributions observed in the CSE frame. The format of the figure is the same as in Fig. 5. Outside the cavity (Figs. 6a-d), the strong fluxes appear in sectors $7^{\prime}$ or $8^{\prime}$ (Figs. 6a,c, d) and 10' (Fig. 6b). Thus, similar to the observations in the CSEQ frame, the accelerated ions flow mainly tailward with cometward/anti-cometward aberrations $\left(\sim 20-40^{\circ}\right)$ in the CSE frame. Inside the cavity cavity (Figs. 6e-h), the flow directions are similar to those outside the cavity in each region. This means that there is a consistent flow pattern between outside and inside of the cavity in the CSE frame. The aberration pattern of the flow is the anti-cometward direction in regions 1,3 , and 4 and the cometward direction in region 2. Thus, near the equator $(X Y)$ plane in the CSE frame, the accelerated ions flow tailward with an anti-cometward aberration both on the $+Y_{\mathrm{CSE}}$ and $-Y_{\mathrm{CSE}}$ sides. On the one hand, near the polar $(X Z)$ plane in the CSE frame, the accelerated ions flow tailward with a cometward aberration on the $+Z_{\mathrm{CSE}}$ side but 


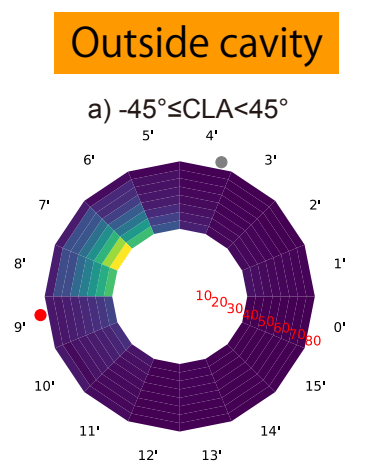

b) $45^{\circ} \leq \mathrm{CLA}<135^{\circ}$

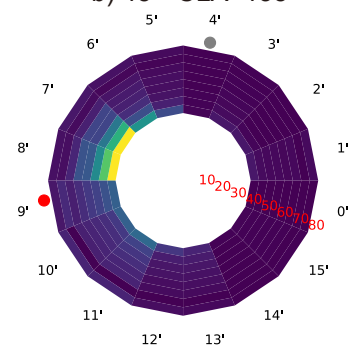

c) $135^{\circ} \leq \mathrm{CLA}<225^{\circ}\left(-135^{\circ}\right)$

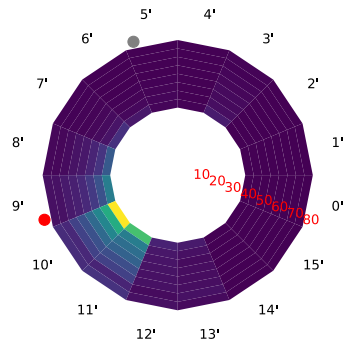

d) $-135^{\circ} \leq \mathrm{CLA}<-45^{\circ}$

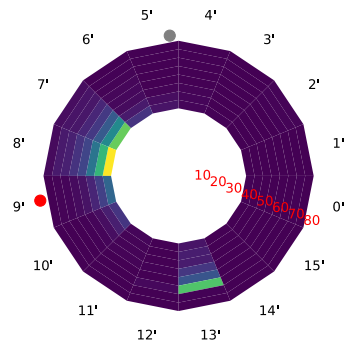

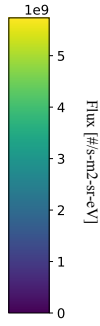
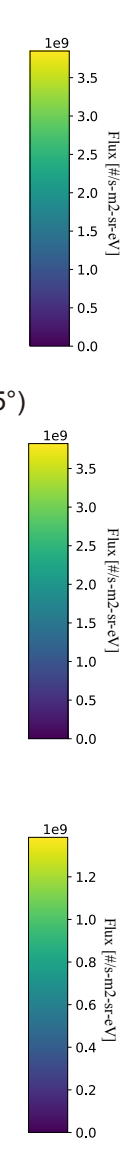

\section{Inside cavity}

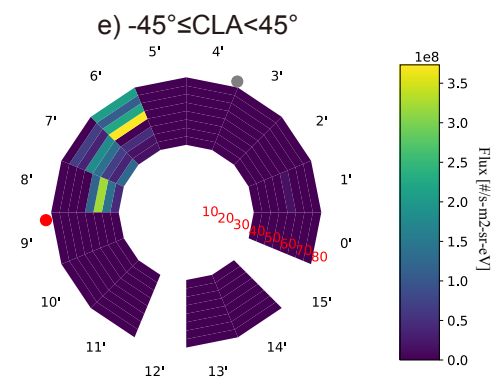

f) $45^{\circ} \leq \mathrm{CLA}<135^{\circ}$

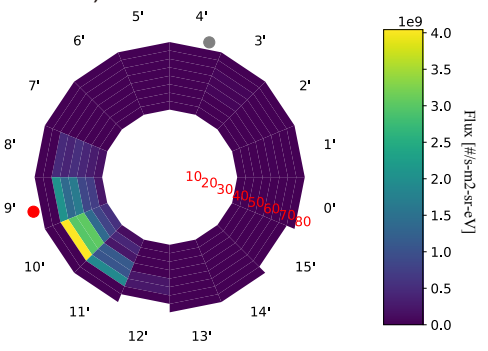

g) $135^{\circ} \leq \mathrm{CLA}<225^{\circ}\left(-135^{\circ}\right)$

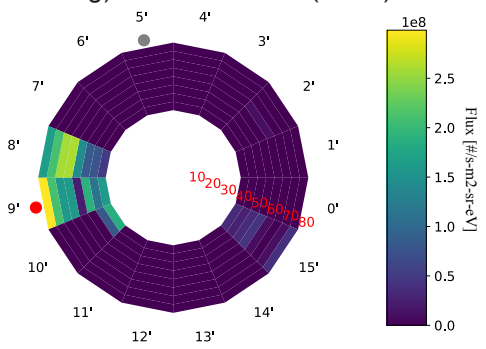

h) $-135^{\circ} \leq \mathrm{CLA}<-45^{\circ}$

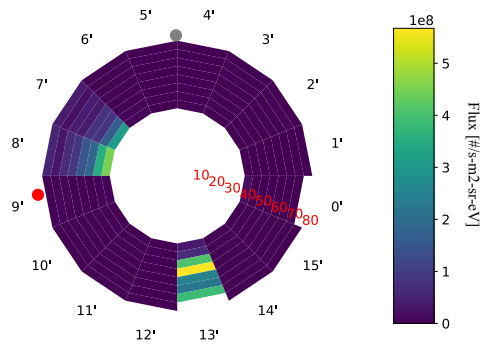

Fig. 5. Average energy-sector flux distributions of the accelerated ions in the CSEQ frame. The plots in the left and right sides are based on observations outside and inside the cavity, respectively. The plots in each row show the different clock angles (CLA) of the Rosetta position in the $Y Z$ plane: $(a$ and $e)-45^{\circ} \leq \mathrm{CLA} \leq 45^{\circ},(b$ and $f) 45^{\circ} \leq \mathrm{CLA} \leq 135^{\circ},(c$ and $g) 135^{\circ} \leq \mathrm{CLA} \leq 225^{\circ}\left(-135^{\circ}\right)$, and $(d$ and $h)-135^{\circ} \leq \mathrm{CLA} \leq-45^{\circ}$. The red numbers shown in the sector $0^{\prime}$ are energy, and the closed red and gray circles by the outer edge of the sector plane indicate the average Sun and comet directions in sector $9^{\prime}$ and sectors $3^{\prime}, 4^{\prime}$, and $5^{\prime}$. White bins indicate no observation caused by unused sectors 0 and 13 of the original sector plane and/or by the energy correction for the cutoff energy.

anti-cometward on the $-Z_{\mathrm{CSE}}$ side. This means that the accelerated ions flow tailward with an aberration in the $-Z_{\mathrm{CSE}}$ direction. The typical energy and flux of the accelerated ions show similar characteristics to those seen in the CSEQ frame. Similar to the CSEQ frame, the typical flux of these ions is nearly one order of magnitude lower inside the cavity than those outside the cavity, except for region 2 .

\section{Discussion}

Our observations show that the accelerated cometary ions $(40-80 \mathrm{eV})$ are ten times more often observed outside the diamagnetic cavity of $67 \mathrm{P}$ than inside of it. This suggests that the accelerated ions mostly flow around the cavity but sometimes penetrate it. Because the accelerated ions are observed intermittently even outside the cavity ( $18 \%$ of the total time), we suggest that these ions are accelerated somewhere upstream of the observation point. Because the accelerated ions mainly flow tailward with an energy of $40-80 \mathrm{eV}$, we suggest that the accelerated ions would have been mainly accelerated by the tailward polarization electric field (Nilsson et al. 2018) rather than the solar wind motional electric field, which causes more energetic ions (several hundred eV) (e.g., Nilsson et al. 2017; Nicolaou et al. 2017). According to Nilsson et al. (2018), the tailward polarization electric field is in the order of $0.1 \mathrm{mV} \mathrm{m}^{-1}$ and thus the accelerated ions with energy of $40-80 \mathrm{eV}$ would 


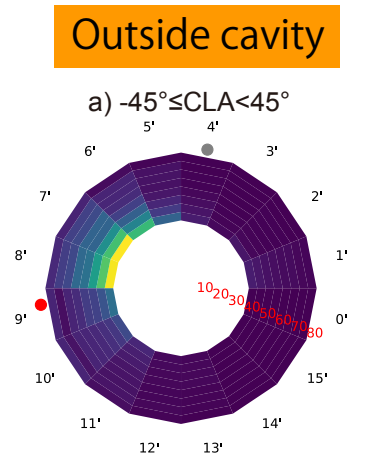

b) $45^{\circ} \leq \mathrm{CLA}<135^{\circ}$

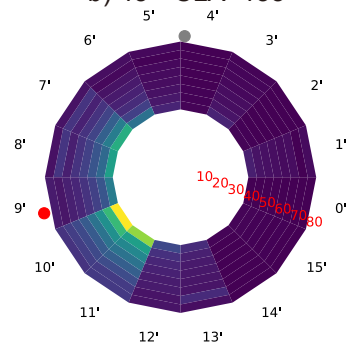

c) $135^{\circ} \leq \mathrm{CLA}<225^{\circ}\left(-135^{\circ}\right)$

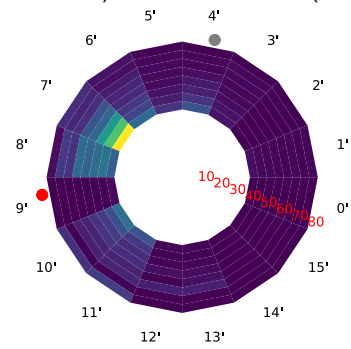

d) $-135^{\circ} \leq \mathrm{CLA}<-45^{\circ}$

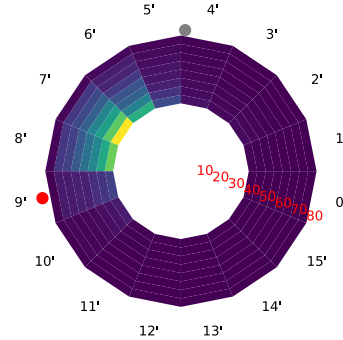

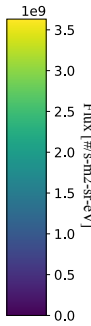
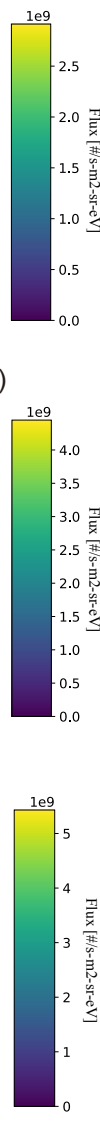

\section{Inside cavity}

e) $-45^{\circ} \leq \mathrm{CLA}<45^{\circ}$
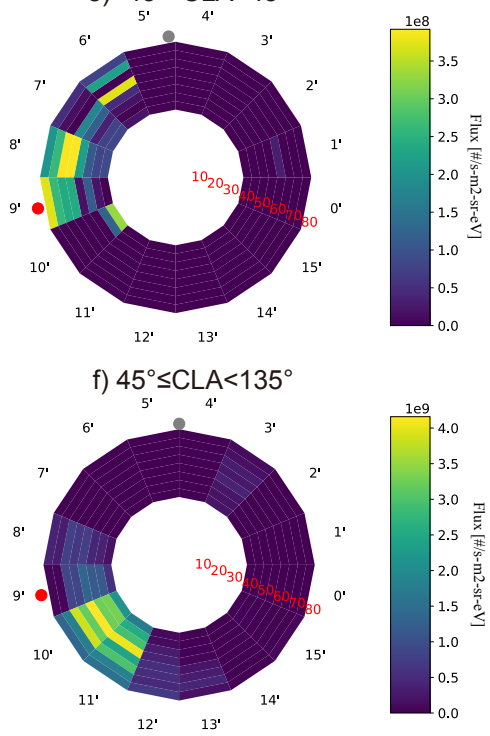

g) $135^{\circ} \leq \mathrm{CLA}<225^{\circ}\left(-135^{\circ}\right)$

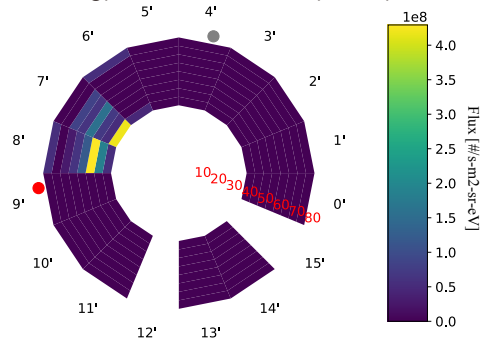

h) $-135^{\circ} \leq \mathrm{CLA}<-45^{\circ}$

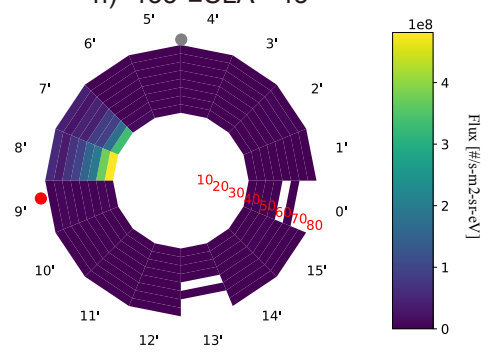

Fig. 6. Average energy-sector flux distributions of the accelerated ions observed outside (left column) and inside (right column) the diamagnetic cavity in the CSE frame. The format is the same as in Fig. 5.

have traveled for $400-800 \mathrm{~km}$ in the electric field. As shown in Tables 1, 2, and 3, the occurrence frequency of the accelerated ions depends on the water production rate at $67 \mathrm{P}$ and becomes lowest near perihelion. This could be caused by the increase in ion-neutral collisions (Mandt et al. 2016) and/or charge exchange (Eviatar et al. 1989) due to large productions and expansion of the neutral gas around the comet near perihelion.

The flow patterns of the accelerated ions inside and outside the diamagnetic cavity of $67 \mathrm{P}$ are illustrated in Fig. 7. The crosses show that the main flow direction of the accelerated ions is tailward. In the CSEQ frame (Fig. 7a), the aberration pattern of the flow is different between inside and outside the cavity, except for region 1. To maintain this flow pattern, there should be some localized effects to accelerate the cometary ions. On the other hand, in the CSE frame (Fig. 7b), the aberration pattern of the flow is the same between inside and outside the diamagnetic cavity in each region. In this frame, the aberration pattern is in the anti-cometward direction near the $X Y$ plane and in the $-Z_{\mathrm{CSE}}$ direction near the $X Z$ plane. Because the ion flow pattern is consistent across the diamagnetic cavity boundary in the CSE frame, we suggest that the flow pattern inside the cavity is determined by the flow outside, depending on the local plasma and magnetic field conditions.

Figure 8 summarizes the ion flow pattern inside and outside the diamagnetic cavity in the CSE frame. The aberration pattern of the accelerated ions in the $-Z_{\mathrm{CSE}}$ direction near the $X Z$ plane seen in Fig. 8a suggests that they are mass-loaded by local cold cometary ions, which is similar to the mass-loading of the solar wind (Behar et al. 2016a,b, 2017). The mass-loading of the accelerated cometary ions would result in an acceleration of the local cold cometary ions in the $+Z_{\mathrm{CSE}}$ direction due to 
a) CSEQ frame

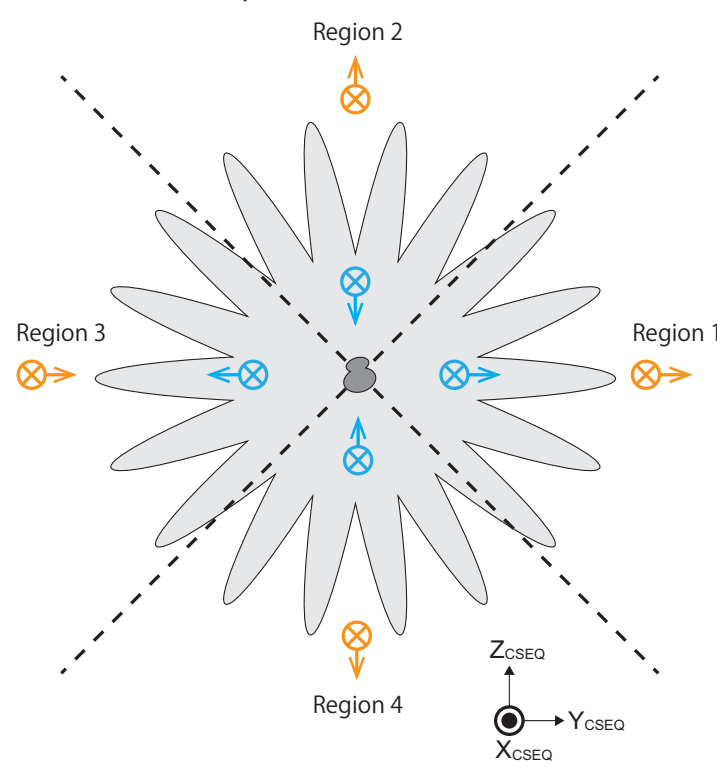

b) CSE frame

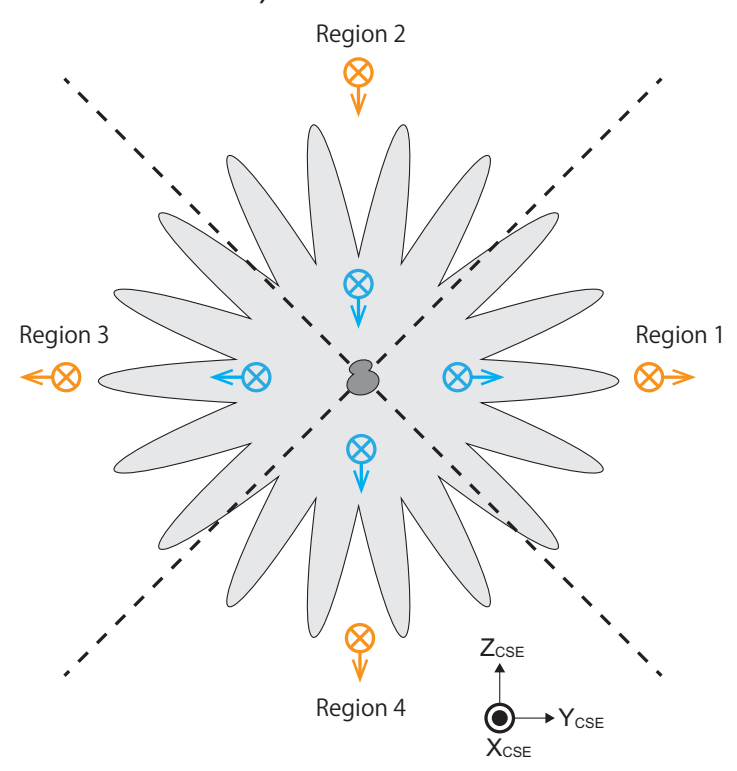

Fig. 7. Summary of flow pattern of the accelerated ions inside and outside the diamagnetic cavity in the $Y Z$ plane of (a) the CSEQ frame and (b) the CSE frame. The dark gray object at the center of each figure indicates the comet nucleus, and the surrounding light gray shaded area is the diamagnetic cavity. The view of these figures is from the Sun, and the four regions depending on the clock angle of the Rosetta position are also shown. In each region, a main tailward flow and its aberration component are shown by a cross and an arrow, respectively.

the conservation of momentum in the system, but we did not detect such a flow pattern from ICA data. This suggests that their energy is very low, which would be out of the energy range in this study $(<40 \mathrm{eV})$, or more likely, the density of the accelerated ions is much lower than the local cometary plasma, meaning that the earning momentum for the cold cometary ions is negligible.

Near the $X Y$ plane of the CSE frame (Fig. 8b), the accelerated ions flow tailward with an anti-cometward aberration. This indicates that the accelerated ions are deflected around the diamagnetic cavity. The mechanism that produces this flow pattern is still unknown, but the accelerated ions may have been deflected when they flew across the upstream boundaries such as the ion-neutral collisionopause or the SWIC boundary. Another possibility is the aforementioned ambipolar electric field that points outward from the nucleus (Odelstad et al. 2018; Berčič et al. 2018). This electric field may cause the tailward-flowing ions to be deflected around the diamagnetic cavity, which results in the anti-cometward aberration of the flow in the $X Y$ plane in the CSE frame.

As seen in observations, the flux of these ions inside the cavity is nearly one order of magnitude lower than that outside the cavity. This suggests that the accelerated ions are only partly transferred from the outside into the inside of the diamagnetic cavity. There may be an energy-filtering effect in the cavity boundary. If the ion transmission into the cavity is more efficient for higher energies, the energy peak could appear at higher energies inside the cavity, but the flux would be lower. We need a numerical simulation that resolves the electric field and currents in the cavity boundary to prove this hypothesis.

Odelstad et al. (2018) showed that ions inside the diamagnetic cavity flow outward from the nucleus with a velocity of 2-4 $\mathrm{km} \mathrm{s}^{-1}$ from LAP and MIP observations. They suggested that the ion motion is not collisionally coupled with neutral particles flowing outward typically with $\sim 1 \mathrm{~km} \mathrm{~s}^{-1}$ and that an outward-pointing ambipolar electric field exists inside the cavity.
We focused on an energy range of $40-80 \mathrm{eV}\left(20-30 \mathrm{~km} \mathrm{~s}^{-1}\right.$ for water ions) in this study, therefore we still have not directly studied the ion flow pattern of the low-energy ions inside and outside the diamagnetic cavity. Because the motion of such low-energy ions is strongly affected by the spacecraft potential, it is difficult to precisely determine the ion motions from ICA data alone. Modeling work is ongoing in our team to study the effect of the spacecraft potential of Rosetta on the motion of the low-energy ions (Bergman et al. 2018).

The role of the accelerated ions in the plasma environment of the comet still remains unknown. However, Rosetta observed the accelerated ions, the increase in magnetic field, and spacecraft potential drops nearly at the same time surrounding the cavity, as shown in Fig. 1. It was shown that the spacecraft potential of Rosetta was mostly negative during the entire mission due to the existence of warm electrons $(\sim 5-10 \mathrm{eV})$ (Odelstad et al. 2015, 2017; Eriksson et al. 2017). The spacecraft potential was mainly controlled by the ambient electron temperature and density (Odelstad et al. 2017). One possibility that might cause the spacecraft potential drop is that the total electron density increased due to the asymmetric steepened features in the magnetic field surrounding the cavity (Henri et al. 2017). Another possibility is that the accelerated ions may contain dense and warm electrons or result in producing them by collisions between the accelerated ions and local cold ions. To investigate these speculations, we plan to study the relationship between the accelerated ions, magnetic field, suprathermal and ambient electrons, and spacecraft potential in a future work.

\section{Conclusion}

We investigated the characteristics of accelerated ions (40$80 \mathrm{eV}$ ) inside and outside the diamagnetic cavity using high time-resolution data from the ICA on board the Rosetta spacecraft. Our main results are listed below. 
a)

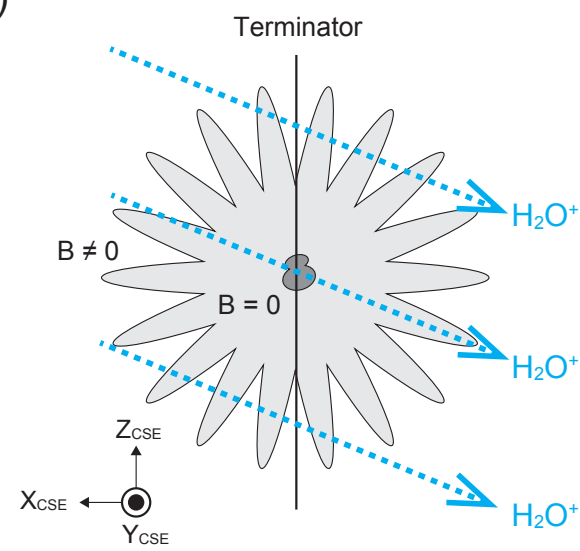

b)

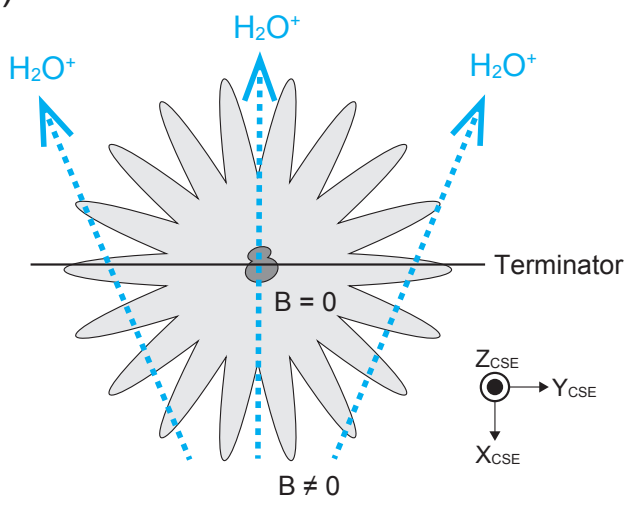

Fig. 8. Summary of the flow pattern of the accelerated cometary ions inside and outside the diamagnetic cavity in $(a)$ the $X Z$ plane and $(b)$ the $X Y$ plane of the CSE frame.

1. The accelerated ions are intermittently observed both inside and outside the diamagnetic cavity. They are nearly ten times more often observed outside the diamagnetic cavity than inside.

2. The occurrence frequency of the accelerated ions become lowest both inside and outside the diamagnetic cavity near perihelion when the water production rate at $67 \mathrm{P}$ was highest.

3. The accelerated ions mainly flow in the tailward direction with an aberration of $\sim 20-40^{\circ}$.

4. The average aberration pattern is different inside and outside the cavity in the CSEQ frame, while it is consistent in the CSE frame.

5. Near the polar $(X Z)$ plane of the CSE frame, the accelerated ions mainly flow tailward with an aberration in the $-Z_{\mathrm{CSE}}$ direction.

6. Near the equator $(X Y)$ plane of the CSE frame, they mainly flow tailward with an anti-cometward aberration.

Based on these results, we suggest that the accelerated ions have been accelerated by the tailward-polarization electric field upstream the comet. They have been accelerated for $400-800 \mathrm{~km}$ in the electric field of $0.1 \mathrm{mV} \mathrm{m}^{-1}$. Ion-neutral collisions and/or charge exchange may play a role in reducing the occurrence frequency of the accelerated ions near perihelion. The aberration pattern of the tailward flow is in the $-Z_{\mathrm{CSE}}$ direction, which suggests that the accelerated ions are mass-loaded by the local cold cometary ions. The cause of the anti-cometward aberration in the $X Y$ plane of the CSE frame is still unknown, but this may indicate that the tailward-flowing cometary ions are deflected across the upstream boundaries or by an outward-pointing ambipolar electric field.

Acknowledgements. This work was supported by the Swedish Research Council under contract 2015-04187. We would like to acknowledge the International Space Science Institute (ISSI) for funding the project 2017-402 "Plasma Environment of Comet 67P after Rosetta". The RPC-MAG data will be made available through the PSA archive of ESA and the PDS archive of NASA. Rosetta is a European Space Agency (ESA) mission with contributions from its member states and the National Aeronautics and Space Administration (NASA).

\section{References}

Balsiger, H., Altwegg, K., Bochsler, P., et al. 2007, Space Sci. Rev., 128, 745 Behar, E., Nilsson, H., Wieser, G. S., et al. 2016a, Geophys. Res. Lett., 43, 1411 Behar, E., Lindkvist, J., Nilsson, H., et al. 2016b, A\&A, 596, A42

Behar, E., Nilsson, H., Alho, M., Goetz, C., \& Tsurutani, B. 2017, MNRAS, 469, S396

Bergman, S., Stenberg Wieser, G., Wieser, M., \& Johansson, F. 2018, Eur. Planet. Sci. Cong., 12, 230

Berčič, L. 2017, In-flight calibration of ICA-cross-talk between azimuth sector anodes, Tech. Rep. 056, Swedish Institute of Space Physics

Berčič, L., Behar, E., Nilsson, H., et al. 2018, A\&A, 613, A57

Biermann, L., Brosowski, B., \& Schmidt, H. U. 1967, Sol. Phys., 1, 254

Carr, C., Cupido, E., Lee, C. G. Y., et al. 2007, Space Sci. Rev., 128, 629

Coates, A. 2004, Adv. Space Res., 33, 1977

Cravens, T. 1987, Adv. Space Res., 7, 147

Edberg, N. J. T., Eriksson, A. I., Vigren, E., et al. 2019, AJ, submitted

Eriksson, A. I., Boström, R., Gill, R., et al. 2007, Space Sci. Rev., 128, 729

Eriksson, A. I., Engelhardt, I. A. D., André, M., et al. 2017, A\&A, 605, A15

Eviatar, A., Goldstein, R., Young, D. T., et al. 1989, ApJ, 339, 545

Glassmeier, K.-H., Boehnhardt, H., Koschny, D., Kührt, E., \& Richter, I. 2007a, Space Sci. Rev., 128, 1

Glassmeier, K.-H., Richter, I., Diedrich, A., et al. 2007b, Space Sci. Rev., 128, 649

Goetz, C., Koenders, C., Richter, I., et al. 2016a, A\&A, 588, A24

Goetz, C., Koenders, C., Hansen, K. C., et al. 2016b, MNRAS, 462, S459

Hajra, R., Henri, P., Vallières, X., et al. 2018, MNRAS, 475, 4140

Hansen, K. C., Altwegg, K., Berthelier, J.-J., et al. 2016, MNRAS, 462, S491

Haser, L. 1957, Bull. Acad. R. de Belgique, Classe de Sci., 43, 740

Henri, P., Vallières, X., Hajra, R., et al. 2017, MNRAS, 469, S372

Ip, W. H., \& Axford, W. I. 1987, Nature, 325, 418 EP

Mandt, K. E., Eriksson, A., Edberg, N. J. T., et al. 2016, MNRAS, 462, S9

Neubauer, F. M., Glassmeier, K. H., Pohl, M., et al. 1986, Nature, 321, 352 EP

Nicolaou, G., Behar, E., Nilsson, H., et al. 2017, MNRAS, 469, S339

Nilsson, H., Lundin, R., Lundin, K., et al. 2007, Space Sci. Rev., 128, 671

Nilsson, H., Stenberg Wieser, G., Behar, E., et al. 2015, Science, 347, 0571

Nilsson, H., Wieser, G. S., Behar, E., et al. 2017, MNRAS, 469, S252

Nilsson, H., Gunell, H., Karlsson, T., et al. 2018, A\&A, 616, A50

Odelstad, E., Eriksson, A. I., Edberg, N. J. T., et al. 2015, Geophys. Res. Lett., 42,126

Odelstad, E., Stenberg-Wieser, G., Wieser, M., et al. 2017, MNRAS, 469, S568

Odelstad, E., Eriksson, A. I., Johansson, F. L., et al. 2018, J. Geophys. Res. Space Phys., 123, 5870

Stenberg Wieser, G., Odelstad, E., Wieser, M., et al. 2017, MNRAS, 469, S522

Szegö, K., Glassmeier, K.-H., Bingham, R., et al. 2000, Space Sci. Rev., 94, 429

Trotignon, J. G., Michau, J. L., Lagoutte, D., et al. 2007, Space Sci. Rev., 128 , 713 